\title{
D-dimer as a stand-alone test to rule out deep vein thrombosis
}

Fridtjof B. Rinde ${ }^{1}$ Synne G. Fronas ${ }^{2,3}$ Waleed Ghanima ${ }^{2,3}$ Anders Vik ${ }^{4}$

John-Bjarne Hansen ${ }^{1,4}$ Sigrid K. Brækkan ${ }^{1,4}$

${ }^{1}$ K.G. Jebsen - Thrombosis Research and Expertise Center (TREC), Department of Clinical Medicine,

UiT - The Arctic University of Norway, Troms $\varnothing$, Norway

${ }^{2}$ Medical division, $\varnothing$ stfold Hospital, Sarpsborg, Norway

${ }^{3}$ Department of Hematology, Institute of Clinical Medicine, University of Oslo, Oslo, Norway

${ }^{4}$ Division of Internal Medicine, University Hospital of North Norway, Troms $\varnothing$, Norway

Short title: D-dimer to rule out DVT

Correspondence to: Fridtjof B. Rinde. K.G. Jebsen - Thrombosis Research and Expertise Center (TREC), Department of Clinical Medicine, UiT - The Arctic University of Norway. E-mail: fridtjof.b.rinde@uit.no.

Word count abstract: 246

Word count text: 3298

Figures: 2 (+ 3 supplementary)

Tables: 3 (+ 1 supplementary)

References: 35

Keyword: clinical prediction rules; D-dimer; deep vein thrombosis; diagnosis; venous thromboembolism. 


\section{Abstract}

Background: Current guidelines recommend the use of clinical decision rules, such as Wells score, in combination with $\mathrm{D}$-dimer to assess the need for objective imaging to rule out deep vein thrombosis (DVT). However, the clinical decision rule has limitations, and use of D-dimer as a stand-alone test has been suggested.

Objective: We aimed to assess the safety and efficiency of D-dimer as a stand-alone test to rule out DVT in outpatients referred with suspected DVT.

Methods: We collected data from consecutive outpatients referred to our hospital with suspected DVT in 2008-2018. D-dimer levels were analyzed using STA ${ }^{\circledast}$ Liatest $^{\circledast}$ D-Di assay. D-dimer as a stand-alone test was theoretically applied in retrospect, and the number of misdiagnosed events were estimated as if such an approach had been initially used. All patients were followed for three months.

Results: Of 1765 included patients, 293 (16.6\%) were diagnosed with DVT. A total of 491 patients (27.8\%) had a negative D-dimer $(<500 \mathrm{ng} / \mathrm{mL})$. Of these, nine were diagnosed with DVT, yielding a failure rate for D-dimer as a stand-alone test of $1.8 \%$ ( $95 \% \mathrm{Cl} 0.8 \%-3.5 \%)$. The majority of the misdiagnosed patients had distal DVT. In analyses restricted to proximal DVTs, the failure rate was $0.6 \%$ (95\% $\mathrm{Cl} 0.1 \%-1.8 \%)$. Ddimer as a stand-alone approach reduced the proportion of required ultrasounds from $81.8 \%$ to $72.2 \%$.

Conclusion: D-dimer as a stand-alone test may be safe for excluding proximal DVT and reduce the proportion of required ultrasounds. Prospective management studies are needed to confirm our findings. 


\section{Highlights}

- D-dimer is proposed as a stand-alone test to rule out deep vein thrombosis (DVT).

- We retrospectively explored the safety and efficiency of such a strategy in a large cohort.

- Our findings suggest that D-dimer as a stand-alone test may be safe for excluding proximal DVT.

- This strategy has the potential to simplify and increase the efficiency of the diagnostic work-up. 


\section{Introduction}

D-dimer is a commonly used biomarker for coagulation activation and fibrinolysis. For patients with a suspected deep vein thrombosis (DVT), current guidelines recommend the use of pretest probability assessment and D-dimer test to evaluate whether DVT can be safely ruled out, or whether the patient should be referred to further diagnostic work-up with imaging techniques [1-3]. The pretest probability assessment is usually performed using Wells score [4], which is a well studied clinical prediction rule for

DVT [5]. The original score was based on signs, symptoms and risk factors for DVT, and classified patients into three risk categories (low, moderate or high probability). A later modification of the Wells score additionally incorporated previous DVT as an item and classifies patients into two categories (unlikely or likely) based on the total score ( $<2$ or $\geq 2$ points) [6]. If the modified Wells score is $<2$ points, D-dimer should be measured, and DVT can be ruled out with a negative predictive value of $98-99 \%$ if the D-dimer level is $<500 \mathrm{ng} / \mathrm{mL}[7]$.

Despite the central role of Wells score in the diagnostic work-up of suspected DVT, the clinical prediction rule has several limitations. The subjective assessment of some of the score items may introduce misclassification [8] and several studies have shown a poor adherence and lack of correct implementation of the score in daily clinical practice [9-12]. As many of today's clinics are organized to be as efficient as possible, standard blood samples including D-dimer are often obtained before clinical evaluation. Consequently, D-dimer is potentially interpreted before calculating the Wells score, contrary to the intention behind the clinical decision rule [13-15].

Although D-dimer is typically increased in patients with acute venous thromboembolism (VTE), D-dimer levels may also be elevated in several other conditions, such as malignancy, infections, and pregnancy, thus decreasing its specificity [16]. Moreover, D-dimer increases with age [17]. Due to a decreased specificity among elderly patients with the fixed cut-off value $(500 \mathrm{ng} / \mathrm{mL})$, age-adjusted cutoff values for D-dimer testing has been suggested [17-20]. 
The use of D-dimer as a stand-alone test to rule out VTE has been proposed, mainly in PE patients [21-23]. A strategy that relies entirely on D-dimer to determine the need for objective imaging may simplify and optimize the efficiency of the diagnostic work-up and reduce the number of imaging tests. In a recent study, Frønæs et al. investigated the safety of D-dimer as a stand-alone test to rule out DVT in 913 outpatients referred with suspected DVT. Of 298 (33\%) patients with a negative D-dimer, only one patient was diagnosed with DVT, yielding a failure rate of $0.3 \%$. These findings indicated that fixed Ddimer as a stand-alone test could safely exclude DVT while requiring fewer compression ultrasounds (CUS) than the combined approach of D-Dimer and Wells score. In the present study, we aimed to validate the findings presented by Frønæs et al. in a larger population of unselected VTE patients. We, therefore, assessed the safety and efficiency of applying D-dimer as a stand-alone test in outpatients consecutively referred to our hospital with suspected DVT. Four diagnostic strategies for excluding DVT were investigated and compared: (i) fixed D-dimer as a stand-alone test, (ii) fixed D-dimer combined with modified Wells score, (iii) age-adjusted D-dimer as a stand-alone test, and (iv) age-adjusted D-dimer combined with modified Wells score. 


\section{Methods}

\section{Study population}

We collected data from consecutive outpatients referred with a suspected DVT to the Emergency Department of the University Hospital of North Norway (UNN), in Troms $\varnothing$, Norway, between 2008 and 2018. The UNN is the sole provider of all VTE diagnostic procedures and VTE-related healthcare in the Troms $\varnothing$ region, serving a local population of 127000 inhabitants. A total of 2003 patients was referred with a suspected DVT during the 10 -year period. Patients with a permanent address outside the catchment area of the hospital $(n=23)$, who could not be followed for three months, were excluded. All included patients were followed for three months after their visit to the Emergency Department by close review of their medical records. Our hospital is the only hospital providing diagnostic and therapeutic management of VTE within a radius of $250 \mathrm{~km}$. Thus, the likelihood of a complete follow-up of patients living in this catchment area is high. Patients in whom the diagnostic work-up was incomplete, i.e. no Ddimer measurement $(n=18)$, no assessment of Wells score $(n=35)$, and patients with an insufficient imaging test $(n=2)$, were excluded. In addition, patients with ongoing anticoagulation treatment were excluded ( $n=160$ ) (Fig. 1). Consequently, 1765 patients were included in the analyses. The study was approved by the regional committee for health and research ethics.

\section{Diagnostic procedure}

All outpatients referred to the Emergency Department with a suspected DVT underwent evaluation by a physician using a modified, two-level Wells score and D-dimer test to guide the decision on further diagnostic testing. The Wells score was assessed in the Emergency Department using a standardized form. D-dimer levels were assayed with the STA ${ }^{\circledR}$-Liatest D-Di from Stago (Diagnostica Stago, Asnieères, France). All blood samples were analyzed at the Department of Clinical Chemistry at the UNN. 
According to existing clinical practice during the data collection period, all patients with either a Wells score $\geq 2$ and/or a positive D-dimer (i.e., $\geq 500 \mathrm{ng} / \mathrm{mL}$ ) at baseline, would be referred for further CUS. However, the physician's clinical judgment could also impact the decision, and some of the patients with negative fixed D-dimer and low clinical probability underwent objective testing, although this was not coherent with the guidelines.

Patients referred for further diagnostic testing underwent a whole-leg CUS, assessing all veins of the affected extremity for compressibility. Non-compressibility was the main criterion for DVT, but a confident gray-scale visualization of the thrombus was also considered diagnostic. In patients where a CUS was impractical (e.g., if leg casting or excessive subcutaneous tissue or fluid prevented adequate assessment of compressibility) or the result of the CUS was uncertain, venography was performed instead or as an additional test.

All patients were followed for three months. In patients where DVT was ruled out at baseline, a diagnosis of DVT occurring in the following three months was considered as an undetected and misclassified event from their first visit. Information on DVT during follow-up was obtained by thorough review of medical records, using the same criteria for objective verification as described above. DVT occurring after the initial three months follow-up period was considered as a new event (i.e., incident or recurrent DVT).

\section{Definition of diagnostic strategies}

Four diagnostic strategies for excluding DVT were investigated (Fig. S1). These included (i) fixed D-dimer as a stand-alone test; (ii) fixed D-dimer combined with modified Wells score; (iii) age-adjusted D-dimer as a stand-alone test; and (iv) age-adjusted D-dimer combined with modified Wells score. In the approaches combining a D-dimer with a modified Wells score, all patients with either a positive D-dimer or a Wells score $\geq 2$ points (i.e., 'DVT likely'), were considered as referred for CUS. When using D-dimer as a stand- 
alone test, patients with a D-dimer below the chosen threshold were considered discharged without further testing. The fixed D-dimer cut-off was defined as positive when the D-dimer value was $\geq 500$ $\mathrm{ng} / \mathrm{mL}$. For the two strategies that included age-adjusted D-dimer, a positive threshold was defined as $\geq$ age $x 10 \mathrm{ng} / \mathrm{mL}$ for patients $\geq 50$ years of age (i.e., the cut-off level for a 70 -year-old would be $\geq 700$ $\mathrm{ng} / \mathrm{mL}$ ). For patients $<50$ years of age, a fixed threshold of $\geq 500 \mathrm{ng} / \mathrm{mL}$ was used.

In our analyses, which were a post hoc estimation of the diagnostic performance of these four different strategies, we theoretically applied the different approaches and used the criteria that would have led to a referral for CUS in each diagnostic strategy. However, the estimations were based on the assumption that the chosen diagnostic criteria would be followed without exception. For instance, while estimating the performance of D-dimer as a stand-alone test, we assumed that all patients with a Ddimer value $<500 \mathrm{ng} / \mathrm{mL}$ would have been discharged without further testing and not treated with anticoagulation.

\section{Statistical analyses}

The failure rate expressed the safety of the strategy and was defined as the proportion of patients who did not meet the criteria for undergoing CUS defined by the chosen strategy (i.e., negative tests), but still was diagnosed with DVT. The amount of required CUS indirectly expressed the efficiency of the approach and was defined as the proportion of patients who met the criteria for undergoing CUS for the chosen strategy (i.e. positive tests).

Since there is an ongoing discussion about the clinical significance of distal DVT with regards to further evaluation and treatment $[24,25]$, we performed a sub-analysis restricted to proximal DVTs. Previous studies have shown that ongoing anticoagulant treatment may influence D-dimer levels and thereby decrease the sensitivity of the test $[26,27]$. In our analyses, patients with ongoing anticoagulant treatment were excluded. The current strategies are based on the rationale that the post-test prevalence 
of DVT might be too high to safely rule out the disease based on a negative D-dimer in patients with a high pretest probability. Therefore, we additionally conducted a sensitivity analysis on the performance of D-dimer restricted to this subgroup of patients with a high pretest probability. Furthermore, as cancer is known to affect D-dimer levels, we performed a sensitivity analysis restricted to patients without cancer [16]. All statistical analyses were performed using Stata version 15.0 (Stata Corporation LP, College Station, TX, USA). 


\section{Results}

Of the 1765 included patients with a suspected DVT, a total of 293 were diagnosed with DVT, yielding an overall prevalence of $16.6 \%$ (95\% Cl 14.9\%-18.4\%). Baseline characteristics are presented in Table 1. Age, sex and duration of symptoms were essentially similar between the two groups. The majority (81\%) of the DVT patients had a Wells score of $\geq 2$ points (i.e. 'DVT likely'), while the non-DVT patients had an approximately equal distribution between 'DVT unlikely' (<2 points) and 'DVT likely' ( $52 \%$ and $48 \%$ respectively).

\section{Fixed D-dimer as a stand-alone test}

Of the 1765 included patients, 491 (27.8\%) had a negative D-dimer and would have been sent home without further testing if D-dimer as a stand-alone test was the chosen diagnostic strategy (Fig. 2). Since this approach was not applied during the study period, 185 were referred for imaging testing despite a negative D-dimer. Of the patients referred for imaging testing, 134 had a Wells score of $\geq 2$ points, while 51 were referred despite both a negative D-dimer and Wells score $<2$ points. Of the 185 patients referred for CUS or venography despite a negative D-dimer, eight were diagnosed with DVT during their first visit to the Emergency Department. In addition, one patient was diagnosed with DVT during the three months follow-up. Of the nine DVTs in patients with a negative D-dimer, six were distal and three were proximal. Of the remaining 306 patients in whom DVT was ruled out due to a negative D-dimer and no CUS was performed, no patient was diagnosed with DVT during the follow-up period. Thus, a total of nine patients with a negative D-dimer were diagnosed with DVT, resulting in a failure rate of $1.8 \%$ (95\% $\mathrm{Cl}$ 0.8-3.5\%) for D-dimer as a stand-alone test (Table 2). D-dimer as a stand-alone test required 1274 referrals $(72.2 \%, 95 \% \mathrm{Cl} 70.0 \%-74.3 \%)$ for CUS. When the modified Wells score was combined with fixed D-dimer, the number of undiagnosed DVTs were reduced to five patients, one proximal, yielding a failure 
rate of $1.6 \%$ (95\% $\mathrm{Cl} 0.5-3.6 \%)$. The combined strategy required 1443 referrals $(81.8 \%, 95 \% \mathrm{Cl} 79.9 \%$ 83.5\%) for CUS (Table 2).

In the sub-analysis restricted to proximal DVT, three of the 491 patients with a negative D-dimer were diagnosed with DVT (Fig. S2), yielding a failure rate of $0.6 \%$ (95\% $\mathrm{Cl} 0.1-1.8 \%$ ) for D-dimer as a stand-alone test (Table 3). In the combined strategy, one patient was diagnosed with a proximal thrombi, and the corresponding failure rate for this strategy was $0.3 \%$ (95\% $0.0-1.7 \%)$.

\section{Age-adjusted D-dimer as a stand-alone test}

Age-adjusted D-dimer as a stand-alone test would have resulted in 13 missed DVTs, corresponding to a failure rate of $2.0 \%(95 \% \mathrm{Cl}$ 1.1\%-3.4\%) (Table 2). Of these 13 patients, nine were diagnosed with a distal thrombus, yielding a failure rate of $0.6 \%(95 \% \mathrm{Cl} 0.2-1.6 \%)$ in the sub-analysis restricted to proximal DVT (Table 3). The modified Wells score in combination with the age-adjusted D-dimer test yielded five undiagnosed DVTs (failure rate $1.2 \%, 95 \% \mathrm{Cl} 0.4-2.9 \%$ ). One of these undiagnosed DVTs was a proximal thrombosis yielding a failure rate of $0.2 \%(95 \% \mathrm{Cl} 0.0-1.4 \%)$ in the analysis restricted to proximal DVT (Table 3).

Sensitivity analyses restricted to patients with high pretest probability and non-cancer patients In our study population, 169 patients with a high pretest probability had a negative D-dimer. Of these 169 patients, four patients were diagnosed with DVT, yielding a failure rate of $2.4 \%(95 \% \mathrm{Cl} 0.6-6.0 \%)$ in this particular subgroup. Two of these four patients were diagnosed with proximal DVT, yielding a failure rate of $1.2 \%(95 \% \mathrm{Cl} 0.1-4.2 \%)$ when restricted to proximal DVTs.

Only one cancer patient with a D-dimer level $<500 \mathrm{ng} / \mathrm{mL}$ was diagnosed with DVT, and the analyses restricted to patients without cancer showed similar results as the main analysis for all approaches, including both fixed and age-adjusted D-dimer (Table S1). 


\section{Discussion}

In the present study, we investigated the safety and efficiency of D-dimer as a stand-alone test for ruling out DVT. With a fixed cut-off value of $500 \mathrm{ng} / \mathrm{mL}$, D-dimer as a stand-alone test performed similarly as Ddimer combined with Wells score (failure rate $1.8 \%$, versus $1.6 \%$ ), but necessitated fewer CUS (72.2\% versus $81.8 \%$ ). The majority of the misclassified patients (i.e., false negative test) had a distal DVT (6/9). Thus, when we restricted our analysis to proximal DVT, the failure rate for D-dimer as a stand-alone test was $0.6 \%$ and the proportion of required CUS was reduced from $81.8 \%$ for the combined strategy to 72.2\% for the stand-alone test. Our findings suggest that D-dimer as a stand-alone test is as safe as Ddimer combined with Wells score to rule out DVT and necessitates $12 \%$ fewer ultrasound examinations.

Frønæs et al. previously evaluated the safety of D-dimer as a stand-alone test in patients with suspected DVT [21]. Among the 913 patients included in their study, 33\% had a negative D-dimer $(<500$ $\mathrm{ng} / \mathrm{mL}$ ), while the prevalence of diagnosed DVT was $18.9 \%$. D-dimer as a stand-alone test yielded a failure rate of $0.3 \%$ while the proportion of required CUS was reduced from $76.9 \%$ (D-dimer combined with Wells score) to $67.4 \%$ (D-dimer as a stand-alone test) [21]. Our study included twice as many patients, and there was no selection of participants in the emergency department. A total of $28 \%$ of the study population had a negative D-dimer, while the prevalence of diagnosed DVT in our study was 16.5\%. The failure rate for $\mathrm{D}$-dimer as a stand-alone test was higher in our study ( $1.8 \%$ versus $0.3 \%)$, but the majority of the "false negative" patients in our study had a distal DVT (67\%). In contrast to the study by Frønæs et al., patients with uncertain CUS findings in our study were referred for contrast venography for further testing. By using this highly sensitive diagnostic test, we would likely detect more distal DVTs than by CUS alone [28]. Consequently, the different practice in objective testing, in addition to different study designs, could partly explain the higher failure rate in our study.

In the present study, the failure rate was lower when we restricted our analyses to proximal DVT. While proximal thrombosis is considered a serious condition with a potentially severe outcome, the 
clinical significance of distal DVT is debated $[1,25]$. In some centers, CUS is only performed in proximal veins and normally repeated after one week in those with an initial negative test to ensure that there is no proximal thrombus extension. With this approach, distal DVTs are not detected and thus left untreated. As our study was a post hoc analysis of current clinical practice, we do not know whether the distal DVTs detected in our study would have progressed or not if they were left untreated. Therefore, our findings should be confirmed in a prospective management study using D-dimer as a stand-alone test.

The failure rate, often considered as the posttest probability in VTE diagnostics, is commonly used to validate diagnostic strategies for VTE $[1,3,29]$. Based on the performance of venography, which is generally accepted as the reference standard within DVT diagnostics, a failure rate estimate of less than $2 \%$ is considered an acceptable degree of safety for a diagnostic pathway [30]. In our study, the failure rate of D-dimer as a stand-alone test for exclusion of all DVTs (including the distal DVTs) was 1.8\%, but the upper limit of the $95 \% \mathrm{Cl}$ exceeded the recommended $2 \%$ limit. However, when restricting the outcome to proximal DVTs, the upper limit of $95 \% \mathrm{Cl}$ was less than $2 \%$. D-dimer as a stand-alone test may therefore be safe to exclude proximal DVTs, but not distal DVTs. In a large individual patient level meta-analysis of more than 10000 patients with suspected DVT, the failure rate for D-dimer in combination with a Wells score $\leq 1$ point was $1.2 \%$ with a $95 \% \mathrm{Cl}$ ranging from $0.7 \%$ to $1.8 \%$ [31]. Thus, the failure rates observed in our study for D-dimer as a stand-alone test to exclude proximal DVT corresponded well with those of current practice. This further supports that D-dimer as a stand-alone test may be safely used to exclude proximal DVT. Finally, the strategy compares satisfactorily to the failure rate observed for CUS, which is estimated to range between $0.6 \%$ and $2.0 \%[32,33]$.

In the subanalysis restricted to patients with a high pretest probability, the failure rate for proximal DVT was $1.2 \%$. Due to limited statistical power of such a small subanalysis $(n=169)$, the point estimate and the following $95 \% \mathrm{Cl}$ should be interpreted with caution. Nevertheless, the point estimate 
for the failure rate of proximal DVT was below the suggested $2 \%$ cut-off even when restricted to those with a high pretest probability.

An age-adjusted cut-off value has been suggested to increase the usefulness of D-dimer among elderly [34]. In our analysis, age-adjusted D-dimer as a stand-alone test would have led to a further reduction in required CUS of $8.9 \%$ percentage points compared to fixed D-dimer as a stand-alone test. However, this approach misdiagnosed additional four DVT patients, yielding a failure rate of $2.0 \%$. While this failure rate is on the $2 \%$ threshold, the fixed D-dimer as a stand-alone test provided a slightly higher degree of safety, and due to its simplicity, one may therefore argue that the fixed D-dimer is a more favorable strategy.

Large campaigns, such as the 'Choosing Wisely' by The American Board of Internal Medicine Foundation, have in recent years increased the focus on unnecessary medical tests and why they should be avoided [35]. One of the main advantages of using D-dimer as a stand-alone test is the reduction of required CUS. Although CUS is a low-risk procedure, the examination is resource demanding. As only a minority of patients with a suspected DVT have the disease, a reduction of CUS could increase the efficiency of the diagnostic work-up [7].

The main strength of our study is the inclusion of consecutive patients with suspected DVT within a confined geographical area for ten subsequent years, yielding a relatively large and unselected study population. Furthermore, the same diagnostic work-up, including the same high sensitive D-dimer assay, was used during the entire study period. Moreover, all diagnostic work-up of VTE in the region is carried out at the UNN, which enhances the likelihood of a complete 3-month follow-up for those living within the catchment area of this hospital. Our study has some limitations that need to be addressed. First, as our study retrospectively applied the diagnostics strategies, the results for the diagnostics strategies are only theoretical estimates of the diagnostic performances. Consequently, clinical aspects in the implementation of $D$-dimer as a stand-alone test are not revealed, and our findings must be further 
validated in a prospective management study. Furthermore, the study only investigates the diagnostic work-up among outpatients, while D-dimer's performance among inpatients is likely to differ [36, 37].

Our findings suggest that D-dimer as a stand-alone test may be safe for excluding proximal DVT in outpatients. This strategy has the potential to simplify and increase the efficiency of the diagnostic work-up for patients with suspected DVT. Since our study was conducted as a post hoc analysis, future prospective management studies are warranted to confirm our findings and investigate the safety of Ddimer as a stand-alone test in outpatients.

Funding

K.G. Jebsen TREC is supported by an independent grant from Stiftelsen K.G. Jebsen.

\section{Conflict of Interest.}

The authors state that they have no conflict of interest. 


\section{Reference}

[1] S.M. Bates, R. Jaeschke, S.M. Stevens, S. Goodacre, P.S. Wells, M.D. Stevenson, C. Kearon, H.J. Schunemann, M. Crowther, S.G. Pauker, R. Makdissi, G.H. Guyatt, Diagnosis of DVT: Antithrombotic Therapy and Prevention of Thrombosis, 9th ed: American College of Chest Physicians Evidence-Based Clinical Practice Guidelines, Chest 141(2 Suppl) (2012) e351S-e418S.

[2] C. National Clinical Guideline, National Institute for Health and Clinical Excellence: Guidance, Venous Thromboembolic Diseases: The Management of Venous Thromboembolic Diseases and the Role of Thrombophilia Testing, Royal College of Physicians (UK)

National Clinical Guideline Centre., London, 2012.

[3] W. Lim, G. Le Gal, S.M. Bates, M. Righini, L.B. Haramati, E. Lang, J.A. Kline, S. Chasteen, M. Snyder, P. Patel, M. Bhatt, P. Patel, C. Braun, H. Begum, W. Wiercioch, H.J. Schunemann, R.A. Mustafa, American Society of Hematology 2018 guidelines for management of venous thromboembolism: diagnosis of venous thromboembolism, Blood Adv 2(22) (2018) 3226-3256.

[4] P.S. Wells, D.R. Anderson, J. Bormanis, F. Guy, M. Mitchell, L. Gray, C. Clement, K.S. Robinson, B. Lewandowski, Value of assessment of pretest probability of deep-vein thrombosis in clinical management, Lancet (London, England) 350(9094) (1997) 1795-8.

[5] N. Sermsathanasawadi, P. Suparatchatpun, T. Pumpuang, K. Hongku, K. Chinsakchai, C. Wongwanit, C. Ruangsetakit, P. Mutirangura, Comparison of clinical prediction scores for the diagnosis of deep vein thrombosis in unselected population of outpatients and inpatients, Phlebology 30(7) (2015) 469-74.

[6] P.S. Wells, D.R. Anderson, M. Rodger, M. Forgie, C. Kearon, J. Dreyer, G. Kovacs, M. Mitchell, B. Lewandowski, M.J. Kovacs, Evaluation of D-dimer in the diagnosis of suspected deep-vein thrombosis, N Engl J Med 349(13) (2003) 1227-35.

[7] P.S. Wells, C. Owen, S. Doucette, D. Fergusson, H. Tran, Does this patient have deep vein thrombosis?, Jama 295(2) (2006) 199-207.

[8] M. Tan, C.J. van Rooden, R.E. Westerbeek, M.V. Huisman, Diagnostic management of clinically suspected acute deep vein thrombosis, Br J Haematol 146(4) (2009) 347-60.

[9] C. Smith, A. Mensah, S. Mal, A. Worster, Is pretest probability assessment on emergency department patients with suspected venous thromboembolism documented before SimpliRED D-dimer testing?, Canadian Journal of Emergency Medicine 10(6) (2008) 519-523.

[10] M. Newnham, H. Stone, R. Summerfield, N. Mustfa, Performance of algorithms and pre-test probability scores is often overlooked in the diagnosis of pulmonary embolism, Bmj 346 (2013) f1557.

[11] P.M. Roy, G. Meyer, B. Vielle, C. Le Gall, F. Verschuren, F. Carpentier, P. Leveau, A. Furber, Appropriateness of diagnostic management and outcomes of suspected pulmonary embolism, Ann Intern Med 144(3) (2006) 157-64.

[12] C.E. Dronkers, M. Tan, G.C. Mol, A. Iglesias Del Sol, M.A. van de Ree, M.V. Huisman, F.A. Klok, Evaluation of the new simple and objective clinical decision rule "I-DVT" in patients with clinically suspected acute deep vein thrombosis, Thrombosis research 141 (2016) 112-8.

[13] N.S. Gibson, R.A. Douma, A. Squizzato, M. Sohne, H.R. Buller, V.E. Gerdes, Application of a decision rule and a D-dimer assay in the diagnosis of pulmonary embolism, Thromb Haemost 103(4) (2010) 84954.

[14] R.A. Douma, J.B. Kessels, H.R. Buller, V.E. Gerdes, Knowledge of the D-dimer test result influences clinical probability assessment of pulmonary embolism, Thrombosis research 126(4) (2010) e271-5. [15] D. Scarvelis, P.S. Wells, Diagnosis and treatment of deep-vein thrombosis, Cmaj 175(9) (2006) 108792.

[16] M. Righini, A. Perrier, P. De Moerloose, H. Bounameaux, D-Dimer for venous thromboembolism diagnosis: 20 years later, J Thromb Haemost 6(7) (2008) 1059-71. 
[17] R.A. Douma, M. Tan, R.E. Schutgens, S.M. Bates, A. Perrier, C. Legnani, D.H. Biesma, J.S. Ginsberg, H. Bounameaux, G. Palareti, M. Carrier, G.C. Mol, G. Le Gal, P.W. Kamphuisen, M. Righini, Using an agedependent $\mathrm{D}$-dimer cut-off value increases the number of older patients in whom deep vein thrombosis can be safely excluded, Haematologica 97(10) (2012) 1507-13.

[18] M. Nybo, A.M. Hvas, Age-adjusted D-dimer cut-off in the diagnostic strategy for deep vein thrombosis: a systematic review, Scand J Clin Lab Invest 77(8) (2017) 568-573.

[19] N. Riva, G. Camporese, M. lotti, E. Bucherini, M. Righini, P.W. Kamphuisen, P. Verhamme, J.D. Douketis, C. Tonello, P. Prandoni, W. Ageno, Age-adjusted D-dimer to rule out deep vein thrombosis: findings from the PALLADIO algorithm, J Thromb Haemost 16(2) (2018) 271-278.

[20] S. Takach Lapner, J.A. Julian, L.A. Linkins, S.M. Bates, C. Kearon, Questioning the use of an ageadjusted D-dimer threshold to exclude venous thromboembolism: analysis of individual patient data from two diagnostic studies, J Thromb Haemost 14(10) (2016) 1953-1959.

[21] S.G. Fronas, H.S. Wik, A. Dahm, C.T. Jorgensen, J. Gleditsch, N. Raouf, F.A. Klok, W. Ghanima, Safety of D-dimer as a stand-alone test for the exclusion of deep vein thrombosis compared to other strategies, J Thromb Haemost (2018).

[22] S.M. Bates, S. Takach Lapner, J.D. Douketis, C. Kearon, J. Julian, S. Parpia, S. Schulman, J.I. Weitz, L.A. Linkins, M. Crowther, W. Lim, F.A. Spencer, A.Y. Lee, P.L. Gross, J. Ginsberg, Rapid quantitative D-dimer to exclude pulmonary embolism: a prospective cohort management study, J Thromb Haemost 14(3) (2016) 504-9.

[23] A. Perrier, S. Desmarais, M.J. Miron, P. de Moerloose, R. Lepage, D. Slosman, D. Didier, P.F. Unger, J.V. Patenaude, H. Bounameaux, Non-invasive diagnosis of venous thromboembolism in outpatients, Lancet (London, England) 353(9148) (1999) 190-5.

[24] C. Kearon, E.A. Akl, A.J. Comerota, P. Prandoni, H. Bounameaux, S.Z. Goldhaber, M.E. Nelson, P.S. Wells, M.K. Gould, F. Dentali, M. Crowther, S.R. Kahn, Antithrombotic therapy for VTE disease:

Antithrombotic Therapy and Prevention of Thrombosis, 9th ed: American College of Chest Physicians Evidence-Based Clinical Practice Guidelines, Chest 141(2 Suppl) (2012) e419S-e496S.

[25] H. Robert-Ebadi, M. Righini, Management of distal deep vein thrombosis, Thrombosis research 149 (2017) 48-55.

[26] F. Couturaud, C. Kearon, S.M. Bates, J.S. Ginsberg, Decrease in sensitivity of D-dimer for acute venous thromboembolism after starting anticoagulant therapy, Blood coagulation \& fibrinolysis : an international journal in haemostasis and thrombosis 13(3) (2002) 241-6.

[27] M. Estivals, H. Pelzer, P. Sie, J. Pichon, H. Boccalon, B. Boneu, Prothrombin fragment $1+2$, thrombinantithrombin III complexes and D-dimers in acute deep vein thrombosis: effects of heparin treatment, $\mathrm{Br}$ J Haematol 78(3) (1991) 421-4.

[28] W.Z. Tomkowski, B.L. Davidson, J. Wisniewska, G. Malek, J. Kober, P. Kuca, B. Burakowska, K. Oniszh, A. Gallus, A.W. Lensing, Accuracy of compression ultrasound in screening for deep venous thrombosis in acutely ill medical patients, Thromb Haemost 97(2) (2007) 191-4.

[29] C. Kearon, Diagnosis of suspected venous thromboembolism, Hematology. American Society of Hematology. Education Program 2016(1) (2016) 397-403.

[30] R. Hull, J. Hirsh, D.L. Sackett, D.W. Taylor, C. Carter, A.G. Turpie, P. Powers, M. Gent, Clinical validity of a negative venogram in patients with clinically suspected venous thrombosis, Circulation 64(3) (1981) 622-5.

[31] G.J. Geersing, N.P. Zuithoff, C. Kearon, D.R. Anderson, A.J. Ten Cate-Hoek, J.L. Elf, S.M. Bates, A.W. Hoes, R.A. Kraaijenhagen, R. Oudega, R.E. Schutgens, S.M. Stevens, S.C. Woller, P.S. Wells, K.G. Moons, Exclusion of deep vein thrombosis using the Wells rule in clinically important subgroups: individual patient data meta-analysis, BMJ 348 (2014) g1340.

[32] N.S. Gibson, S.M. Schellong, D.Y. Kheir, J. Beyer-Westendorf, A.S. Gallus, S. McRae, R.E. Schutgens, F. Piovella, V.E. Gerdes, H.R. Buller, Safety and sensitivity of two ultrasound strategies in patients with 
clinically suspected deep venous thrombosis: a prospective management study, J Thromb Haemost 7(12) (2009) 2035-41.

[33] S.A. Johnson, S.M. Stevens, S.C. Woller, E. Lake, M. Donadini, J. Cheng, J. Labarere, J.D. Douketis, Risk of deep vein thrombosis following a single negative whole-leg compression ultrasound: a systematic review and meta-analysis, Jama 303(5) (2010) 438-45.

[34] R.A. Douma, G. le Gal, M. Söhne, M. Righini, P.W. Kamphuisen, A. Perrier, M.J.H.A. Kruip, H.

Bounameaux, H.R. Büller, P.-M. Roy, Potential of an age adjusted D-dimer cut-off value to improve the exclusion of pulmonary embolism in older patients: a retrospective analysis of three large cohorts, BMJ 340 (2010).

[35] B. Hudzik, M. Hudzik, L. Polonski, Choosing wisely: avoiding too much medicine, Canadian family physician Medecin de famille canadien 60(10) (2014) 873-6, 884-7.

[36] D.J. Brotman, J.B. Segal, J.T. Jani, B.G. Petty, T.S. Kickler, Limitations of D-dimer testing in unselected inpatients with suspected venous thromboembolism, The American journal of medicine $114(4)(2003)$ 276-82.

[37] J. Thachil, D.A. Fitzmaurice, C.H. Toh, Appropriate use of D-dimer in hospital patients, The American journal of medicine 123(1) (2010) 17-9. 
Figures

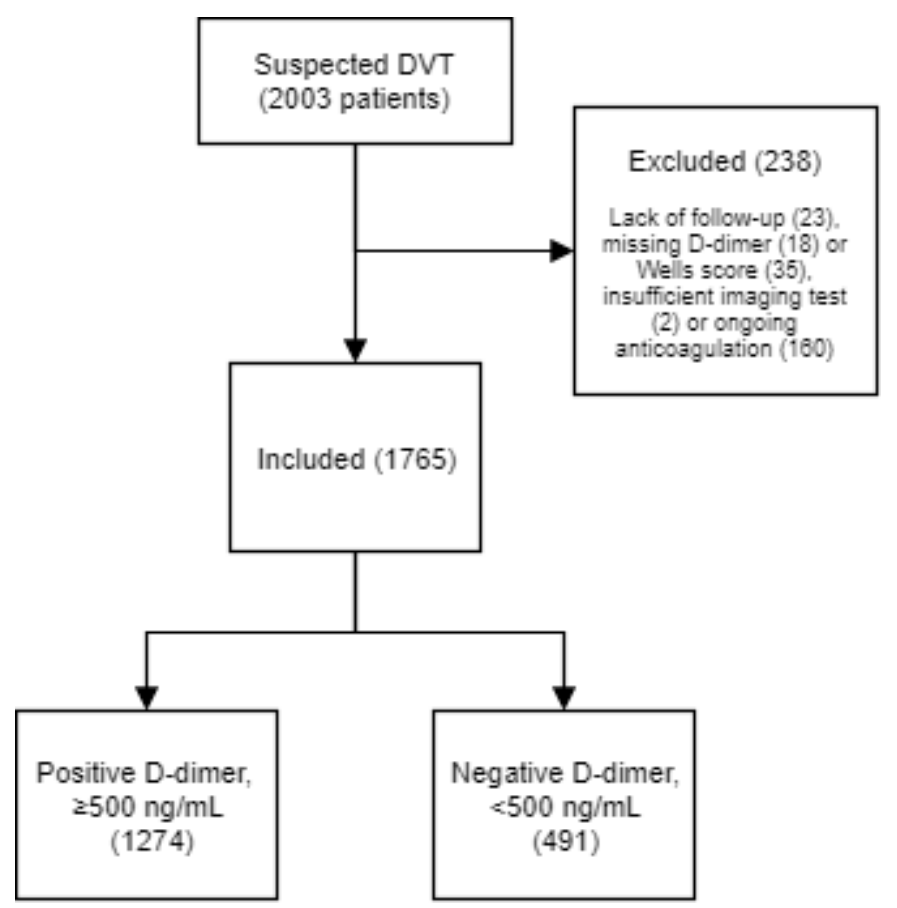

Fig. 1 Study population and design.

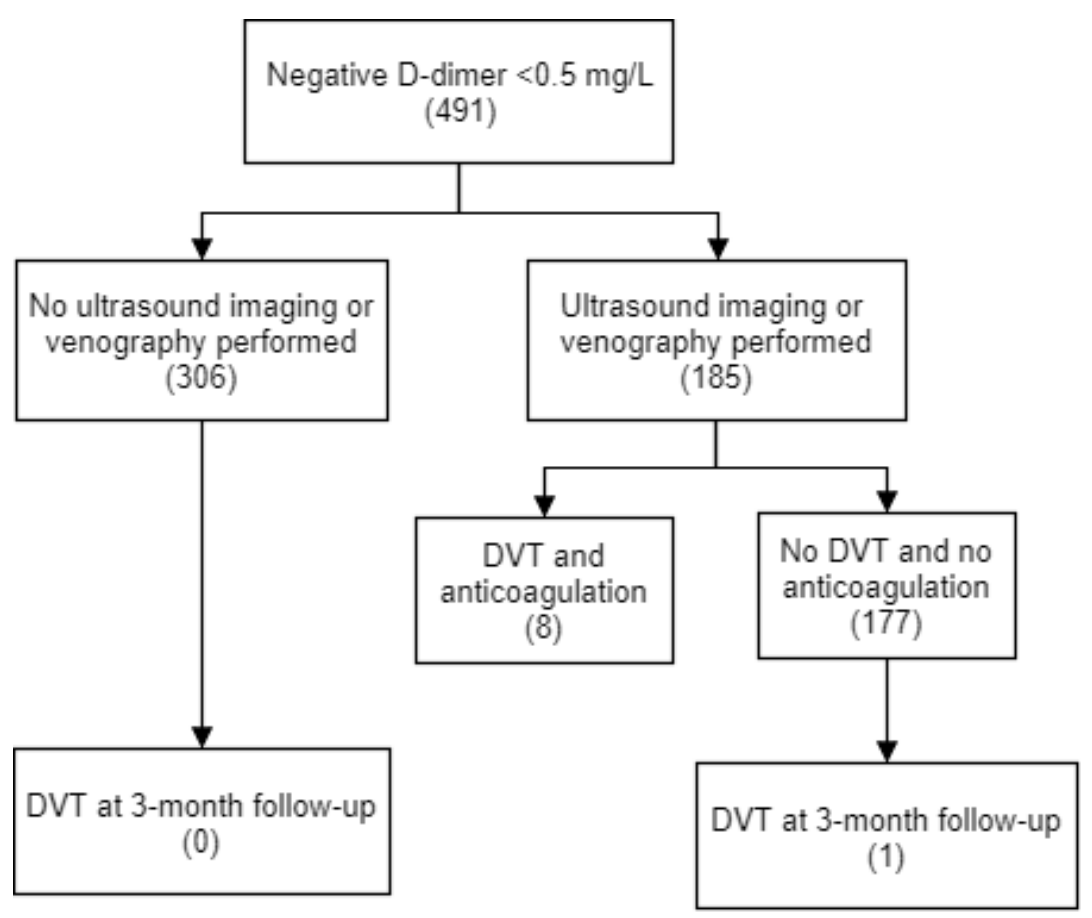




\section{Tables}

Table 1 Demographics and patients characteristics.

\begin{tabular}{llll}
\hline & All & DVT & No DVT \\
& $\mathrm{n}=1765$ & $\mathrm{n}=293$ & $\mathrm{n}=1472$ \\
\hline Age, years, median (IQR) & $63(28)$ & $65(29)$ & $62(27)$ \\
Symptoms duration, days, median (IQR) & $5(12)$ & $5(11)$ & $5(12)$ \\
Female sex, $\mathrm{n}(\%)$ & $969(55)$ & $141(48)$ & $828(56)$ \\
Modified Wells score DVT likely, $\mathrm{n}(\%)$ & $948(54)$ & $237(81)$ & $761(52)$ \\
Modified Wells score DVT unlikely, $\mathrm{n}(\%)$ & $817(46)$ & $56(19)$ & $179(13)$ \\
Previous DVT, $\mathrm{n}$ (\%) & $252(15)$ & $73(27)$ & $53(4)$ \\
Active cancer within past 6 months, $\mathrm{n}(\%)$ & $77(4)$ & $24(8)$ & \\
\hline
\end{tabular}

Abbreviations: IQR, interquartile range; DVT, deep vein thrombosis 
Table 2 Diagnostic performance of the different strategies $(n=1765)$

\begin{tabular}{|c|c|c|c|c|}
\hline & Fixed D-dimer & with Wells score $\geq 2$ & Age-adjusted D-dimer & with Wells score $\geq 2$ \\
\hline \multicolumn{5}{|l|}{ Sensitivity } \\
\hline $\mathrm{TP} /(\mathrm{TP}+\mathrm{FN})$ & $284 / 293$ & $288 / 293$ & $280 / 293$ & $288 / 293$ \\
\hline Estimate,\% & $96.9 \%$ & $98.3 \%$ & $95.6 \%$ & $98.3 \%$ \\
\hline $95 \% \mathrm{Cl}$ & $94.2-98.6$ & $96.1-99.4$ & $92.5-97.6$ & $96.1-99.4$ \\
\hline \multicolumn{5}{|l|}{ Specificity } \\
\hline $\mathrm{TN} /(\mathrm{TN}+\mathrm{FP})$ & $482 / 1472$ & $317 / 1472$ & $635 / 1472$ & $397 / 1472$ \\
\hline Estimate, \% & $32.7 \%$ & $21.5 \%$ & $43.1 \%$ & $27.0 \%$ \\
\hline $95 \% \mathrm{Cl}$ & $30.3-35.2$ & $19.5-23.7$ & $40.6-45.7$ & $24.7-29.3$ \\
\hline \multicolumn{5}{|l|}{ Negative predictive value } \\
\hline $\mathrm{TN} /(\mathrm{TN}+\mathrm{FN})$ & $482 / 491$ & $317 / 322$ & $635 / 648$ & $397 / 402$ \\
\hline Estimate, \% & $98.2 \%$ & $98.4 \%$ & $98.0 \%$ & $98.8 \%$ \\
\hline $95 \% \mathrm{Cl}$ & $96.5-99.2$ & $96.4-99.5$ & 96.6-98.9 & 97.1-99.6 \\
\hline \multicolumn{5}{|l|}{ Positive predictive value } \\
\hline $\mathrm{TP} /(\mathrm{TP}+\mathrm{FP})$ & $284 / 1274$ & $288 / 1443$ & $280 / 1117$ & $288 / 1363$ \\
\hline Estimate, \% & $22.3 \%$ & $20.0 \%$ & $25.1 \%$ & $21.1 \%$ \\
\hline $95 \% \mathrm{Cl}$ & $20.0-24.7$ & $17.9-22.1$ & $22.5-27.7$ & $19.0-23.4$ \\
\hline \multicolumn{5}{|l|}{ Failure rate } \\
\hline $\mathrm{FN} /(\mathrm{FN}+\mathrm{TN})$ & $9 / 491$ & $5 / 322$ & $13 / 648$ & $5 / 402$ \\
\hline Estimate, \% & $1.8 \%$ & $1.6 \%$ & $2.0 \%$ & $1.2 \%$ \\
\hline $95 \% \mathrm{Cl}$ & $0.8-3.5$ & $0.5-3.6$ & $1.1-3.4$ & $0.4-2.9$ \\
\hline \multicolumn{5}{|l|}{ Required CUS } \\
\hline$(T P+F P) /(T P+F N+F P+T N)$ & $1274 / 1765$ & $1443 / 1765$ & $1117 / 1765$ & $1363 / 1765$ \\
\hline Estimate, \% & $72.2 \%$ & $81.8 \%$ & $63.3 \%$ & $77.2 \%$ \\
\hline $95 \% \mathrm{Cl}$ & 70.0-74.3 & 79.9-83.5 & 61.0-65.5 & $75.2-79.2$ \\
\hline
\end{tabular}

Abbreviations: $\mathrm{Cl}$, confidence interval; TP, true positive; TN, true negative; FP, false positive; FN, false negative; CUS, compression ultrasound 
Table 3 Diagnostic performance of the different strategies for exclusion of proximal DVT ( $n=1765$ )

\begin{tabular}{|c|c|c|c|c|}
\hline & Fixed D-dimer & with Wells score $\geq 2$ & Age-adjusted D-dimer & with Wells score $\geq 2$ \\
\hline \multicolumn{5}{|l|}{ Sensitivity } \\
\hline $\mathrm{TP} /(\mathrm{TP}+\mathrm{FN})$ & $185 / 188$ & $187 / 188$ & $184 / 188$ & $187 / 188$ \\
\hline Estimate, \% & $98.4 \%$ & $99.5 \%$ & $97.9 \%$ & $99.5 \%$ \\
\hline $95 \% \mathrm{Cl}$ & $95.4-99.7$ & $97.1-100.0$ & 94.6-99.4 & $97.1-100.0$ \\
\hline \multicolumn{5}{|l|}{ Specificity } \\
\hline $\mathrm{TN} /(\mathrm{TN}+\mathrm{FP})$ & $488 / 1577$ & $321 / 1577$ & $644 / 1577$ & $401 / 1577$ \\
\hline Estimate, \% & $30.9 \%$ & $20.4 \%$ & $40.8 \%$ & $25.4 \%$ \\
\hline $95 \% \mathrm{Cl}$ & 28.7-33.3 & $18.4-22.4$ & $38.4-43.3$ & $23.3-27.7$ \\
\hline \multicolumn{5}{|l|}{ Negative predictive value } \\
\hline $\mathrm{TN} /(\mathrm{TN}+\mathrm{FN})$ & $488 / 491$ & $321 / 322$ & $644 / 648$ & $401 / 402$ \\
\hline Estimate, \% & $99.4 \%$ & $99.7 \%$ & $99.4 \%$ & $99.8 \%$ \\
\hline $95 \% \mathrm{Cl}$ & $98.2-99.9$ & $98.3-100.0$ & 98.4-99.8 & $98.6-100.0$ \\
\hline \multicolumn{5}{|l|}{ Positive predictive value } \\
\hline$T P /(T P+F P)$ & $185 / 1274$ & $187 / 1443$ & $184 / 1117$ & $187 / 1363$ \\
\hline Estimate, \% & $14.5 \%$ & $13.0 \%$ & $16.5 \%$ & $13.7 \%$ \\
\hline $95 \% \mathrm{Cl}$ & $12.6-16.6$ & $11.3-14.8$ & $14.3-18.8$ & $11.9-15.7$ \\
\hline \multicolumn{5}{|l|}{ Failure rate } \\
\hline $\mathrm{FN} /(\mathrm{FN}+\mathrm{TN})$ & $3 / 491$ & $1 / 322$ & $4 / 648$ & $1 / 402$ \\
\hline Estimate, \% & $0.6 \%$ & $0.3 \%$ & $0.6 \%$ & $0.2 \%$ \\
\hline $95 \% \mathrm{Cl}$ & $0.1-1.8$ & $0.0-1.7$ & $0.2-1.6$ & $0.0-1.4$ \\
\hline \multicolumn{5}{|l|}{ Required CUS } \\
\hline$(T P+F P) /(T P+F N+F P+T N)$ & $1274 / 1765$ & $1443 / 1765$ & $1117 / 1765$ & $1363 / 1765$ \\
\hline Estimate, \% & $72.2 \%$ & $81.8 \%$ & $63.3 \%$ & $77.2 \%$ \\
\hline $95 \% \mathrm{Cl}$ & $70.0-74.3$ & 79.9-83.5 & $61.0-65.5$ & $75.2-79.2$ \\
\hline
\end{tabular}

Abbreviations: $\mathrm{Cl}$, confidence interval; TP, true positive; TN, true negative; FP, false positive; FN, false negative; CUS, compression ultrasound 


\section{Supplementary}

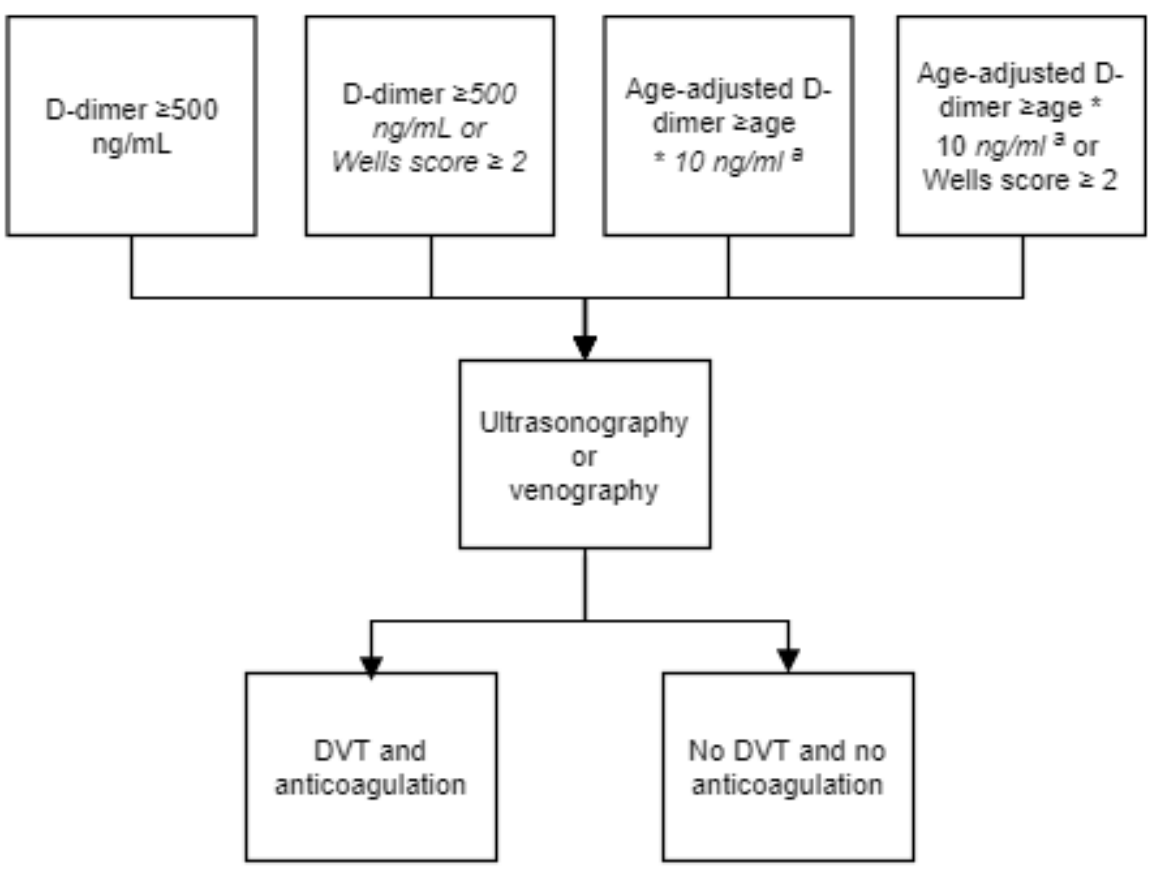

Fig. S1 Strategies in the diagnostic work-up of DVT.

alf age $\geq 50$, otherwise D-dimer $500 \mathrm{ng} / \mathrm{mL}$ 


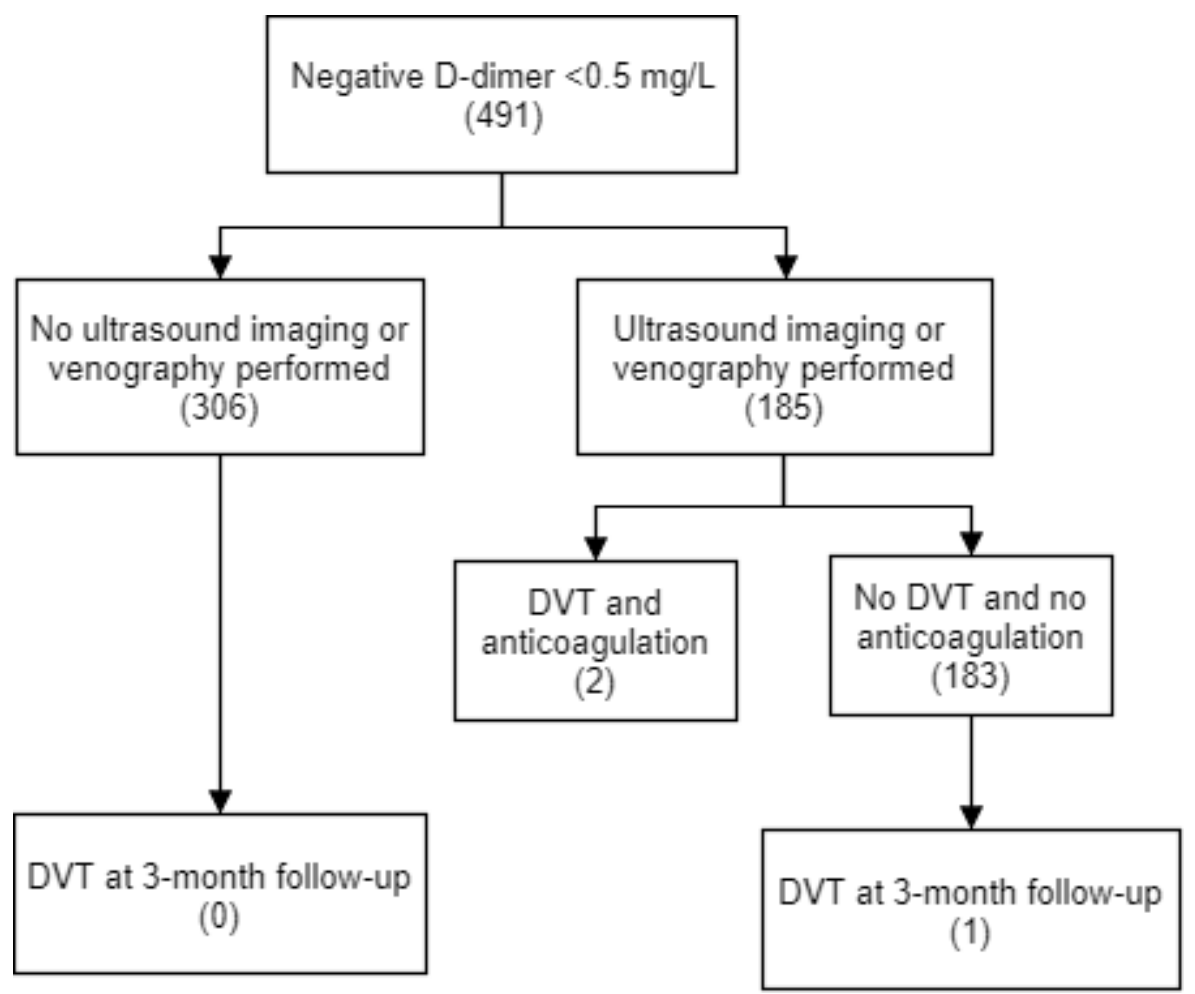

Fig. S2 Fixed D-dimer as a stand-alone test for excluding proximal deep vein thrombosis 


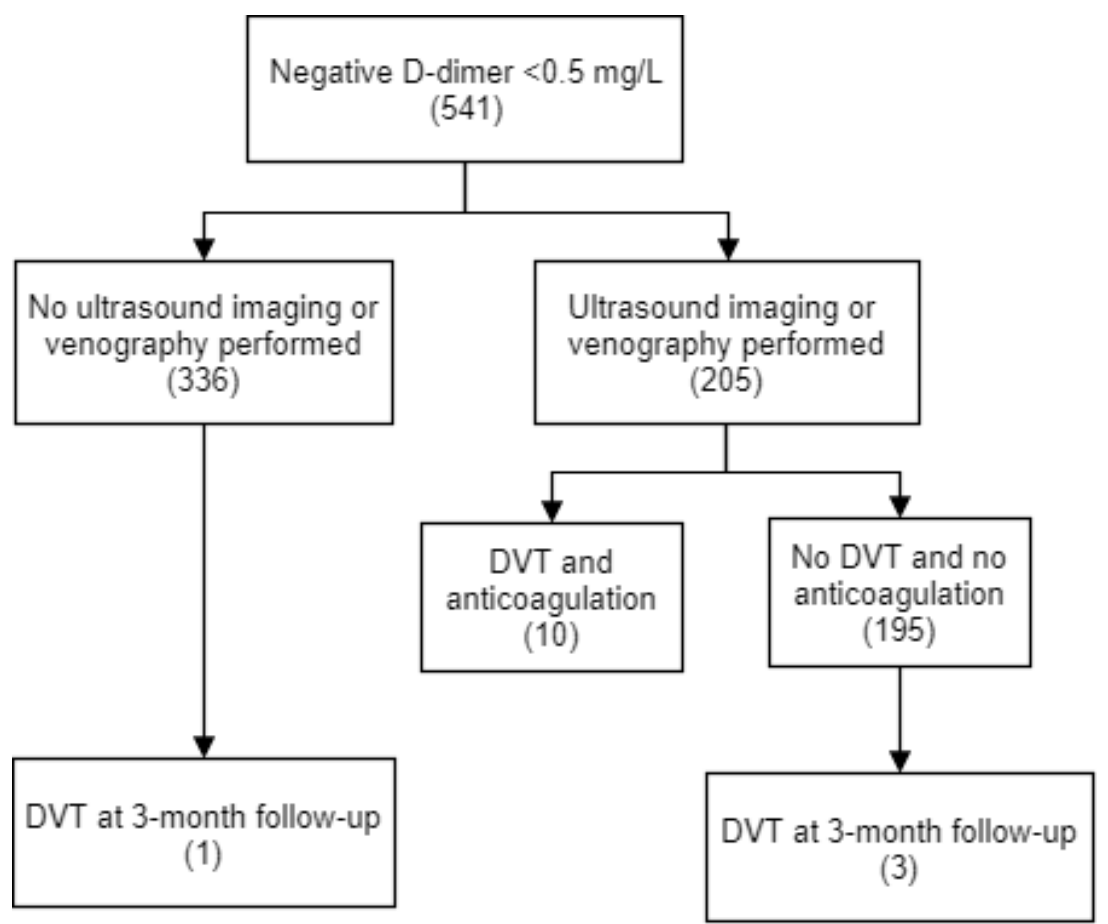

Fig. S3 Fixed D-dimer as a stand-alone test for excluding deep vein thrombosis, including all patients on ongoing anticoagulation treatment. 
Table S1 Diagnostic performance of different strategies after exclusion of cancer patients $(n=1688)$

\begin{tabular}{|c|c|c|c|c|}
\hline & Fixed D-dimer & with Wells score $\geq 2$ & Age-adjusted D-dimer & with Wells score $\geq 2$ \\
\hline \multicolumn{5}{|l|}{ Sensitivity } \\
\hline $\mathrm{TP} /(\mathrm{TP}+\mathrm{FN})$ & $261 / 269$ & $264 / 269$ & $257 / 269$ & $264 / 269$ \\
\hline Estimate, \% & $97.0 \%$ & $98.1 \%$ & $95.5 \%$ & $98.1 \%$ \\
\hline $95 \% \mathrm{Cl}$ & $94.2-98.7$ & $95.7-99.4$ & 92.3-97.7 & $95.7-99.4$ \\
\hline \multicolumn{5}{|l|}{ Specificity } \\
\hline $\mathrm{TN} /(\mathrm{TN}+\mathrm{FP})$ & $470 / 1419$ & $312 / 1419$ & $618 / 1419$ & $390 / 1419$ \\
\hline Estimate, \% & $33.1 \%$ & $22.0 \%$ & $43.6 \%$ & $27.5 \%$ \\
\hline $95 \% \mathrm{Cl}$ & $30.7-35.6$ & $19.9-24.2$ & $41.0-46.2$ & $25.2-29.9$ \\
\hline \multicolumn{5}{|l|}{ Negative predictive value } \\
\hline $\mathrm{TN} /(\mathrm{TN}+\mathrm{FN})$ & $470 / 478$ & $312 / 317$ & $618 / 630$ & $390 / 395$ \\
\hline Estimate, \% & $98.3 \%$ & $98.4 \%$ & $98.1 \%$ & $98.7 \%$ \\
\hline $95 \% \mathrm{Cl}$ & $96.7-99.3$ & $96.4-99.5$ & $96.7-99.0$ & $97.1-99.6$ \\
\hline \multicolumn{5}{|l|}{ Positive predictive value } \\
\hline$T P /(T P+F P)$ & $261 / 1210$ & $264 / 1371$ & $257 / 1058$ & $264 / 1293$ \\
\hline Estimate, \% & $21.6 \%$ & $19.3 \%$ & $24.3 \%$ & $20.4 \%$ \\
\hline $95 \% \mathrm{Cl}$ & $19.3-24.0$ & $17.2-21.4$ & $21.7-27.0$ & $18.3-22.7$ \\
\hline \multicolumn{5}{|l|}{ Failure rate } \\
\hline $\mathrm{FN} /(\mathrm{FN}+\mathrm{TN})$ & $8 / 478$ & $5 / 317$ & $12 / 630$ & $5 / 395$ \\
\hline Estimate, \% & $1.7 \%$ & $1.6 \%$ & $1.9 \%$ & $1.3 \%$ \\
\hline $95 \% \mathrm{Cl}$ & $0.7-3.3$ & $0.5-3.6$ & $1.0-3.3$ & $0.4-2.9$ \\
\hline \multicolumn{5}{|l|}{ Required CUS } \\
\hline$(T P+F P) /(T P+F N+F P+T N)$ & $1210 / 1688$ & $1371 / 1688$ & $1058 / 1688$ & $1293 / 1688$ \\
\hline Estimate, \% & $71.7 \%$ & $81.2 \%$ & $62.7 \%$ & $76.6 \%$ \\
\hline $95 \% \mathrm{Cl}$ & $69.5-73.8$ & $79.3-83.1$ & $60.3-65.0$ & $74.5-78.6$ \\
\hline
\end{tabular}

Abbreviations: $\mathrm{Cl}$, confidence interval; $\mathrm{TP}$, true positive; TN, true negative; $\mathrm{FP}$, false positive; $\mathrm{FN}$, false negative; CUS, compression ultrasound 